\title{
Klippel-Trenaunay Syndrome (KTS)
}

National Institute of Neurological Disorders and Stroke (NINDS)

\section{Source}

National Institute of Neurological Disorders and Stroke (NINDS). Klippel-Trenaunay

Syndrome (KTS) Information Page.

Klippel-Trenaunay syndrome (KTS) is a rare congenital malformation involving blood and lymph vessels and abnormal growth of soft and bone tissue. Typical symptoms include hemangiomas (abnormal benign growths on the skin consisting of masses of blood vessels) and varicose veins. Fused toes or fingers, or extra toes or fingers, may be present. In some cases, internal bleeding may occur as a result of blood vessel malformations involving organs such as the stomach, rectum, vagina, liver, spleen, bladder, kidneys, lungs, or heart. Individuals are also at risk for blood clots. The cause of the disorder is unknown. A similar port-wine stain disorder in which individuals have vascular anomalies on the face as well as in the brain is Sturge-Weber syndrome. These individuals may experience seizures and mental deficiency. In some cases, features of the Klippel-T renaunay syndrome and Sturge-Weber syndrome coincide. Another overlapping condition is the Parkes-Weber syndrome, which is characterized by abnormal connectivity between the arterial and venous system (arteriovenous fistulas). 